\title{
Modelling polyurethane synthesis rates on the example of heteropolyaddition of 2,4-toluene diisocyanate (TDI) and 1,4-butanediol (BD)
}

\section{Tadeusz Mederski ${ }^{1}$ (B)}

Received: 16 January 2018 / Revised: 7 August 2018 / Accepted: 20 August 2018 / Published online: 29 August 2018 (c) The Author(s) 2018

\begin{abstract}
This paper investigates the effects of increase of reaction mixture viscosity on the kinetics of linear polymer creation in a bulk heteropolyaddition process of 2,4-toluene diisocyanate (TDI) and 1,4-butanediol (BD). The paper presents a method for solving a system of bulk polyaddition of 2,4-toluene diisocyanate and 1,4-butanediol process balance equations, allowing the determination of the process kinetic parameters. Determination of polymerisation reaction kinetic parameters was also made possible by the use of the so-called partial reaction rate constant. Such an approach enabled a significant simplification of the mathematical expressions describing the heteropolyaddition process and provided an opportunity to associate kinetic parameters with the average molar mass of the mixture and, thus, with the viscosity. The method presented herein facilitates an analysis of the linear polymers heteropolyaddition process.
\end{abstract}

Keywords Kinetics of polymerisations - Reaction rate constant - Linear polymer · Viscosity $\cdot$ Heteropolyaddition $\cdot$ Modelling of polymerisations

\section{List of symbols}

$E_{i} \quad$ Experimental value (literature), [g/mol]

$k_{1} \quad$ Partial reaction rate constant of component $1,\left[\mathrm{dm}^{3} /(\mathrm{mol} \mathrm{s})^{1 / 2}\right]$

$k_{\mathrm{A}}, k_{\mathrm{B}}$ Partial reaction rate constants of reagents assigned to reagents $\mathrm{A}$ and $\mathrm{B}$, respectively, $\left[\mathrm{dm}^{3} /(\mathrm{mol} \mathrm{s})^{1 / 2}\right]$

$k_{\mathrm{AB}} \quad$ Reaction rate constant $\mathrm{A}+\mathrm{B}=\mathrm{AB},\left[\mathrm{dm}^{3} /(\mathrm{mol} \mathrm{s})\right]$

$k_{j} \quad$ Partial reaction rate constant $j$ of this component, $\left[\mathrm{dm}^{3} /(\mathrm{mol} \mathrm{s})^{1 / 2}\right]$

$M_{0} \quad$ Molar mass of monomer A or B, [g/mol]

$M_{n} \quad$ Polymer number-average molar mass, [g/mol]

$O_{i} \quad$ Calculated value, $[\mathrm{g} / \mathrm{mol}]$

Tadeusz Mederski

tadeusz@uni.opole.pl

1 Independent Department of Process Engineering, Opole University, R. Dmowski street no. 7-9, 45-365 Opole, Poland 


$\begin{array}{ll}t & \text { Time [min] } \\ w_{i} & \text { Part by weight } \\ Z_{i} & \text { Correction coefficient of component } i \\ \beta(\eta) & \text { Correlation coefficient, } \beta(\eta) \in(0.1) \\ \gamma & \text { Constant coefficient characteristic for a particular polymerisation process }\end{array}$

\section{Introduction}

Very often during the course of polymerisation processes, the viscosity of the reaction medium changes. When these changes significantly influence the polymerisation process rate, it becomes necessary to take into account these variables in balance equations describing this process. The need to take into account changes in viscosity means that systems of mass balance equations describing the polymerisation process are usually quite complex and often difficult to solve due to the large number of unknown kinetic parameters. In connection with difficulties in determining polymerisation process kinetic parameters, literature often assumes the same value for all constant rates of these reactions $(k=\mathrm{const}$ ), and the effect of viscosity changes on the numerical values of the $k$ constants is ignored. Used method is appropriate for systems, where viscosity is practically constant.

This paper attempts to take into account increasing viscosity of the reaction medium on the kinetics of the linear polymer creation process occurring during heteropolyaddition process. The increase in average molar mass of the mixture and, thus, the viscosity caused by the course of this process makes the constant reaction rate numerical values decrease and as such may have a significant effect on the results.

Due to the complexity of the mass balance equation system and the necessity to take into account viscosity changes in numerical calculations, this paper uses the concept of partial reaction rate constants, the introduction of which into the balance equations allows a meaningful simplification of the mathematical model describing the polyaddition process being considered here [1]. The influence of viscosity on the polymerisation rate has been taken into account by associating that rate with the number-average molecular mass $M_{n}$ of the reaction mixture, which provided an opportunity to associate kinetic parameters $k$ with the number-average molar mass $M_{n}$. Using the derived mass balance system of equations, which takes into account number-average molar mass changes on the reaction rate constant numerical values, kinetic parameter values for the analysed processes were determined.

The presented method facilitates determination of partial reaction rate constant numerical values and, thus, it assists the progress of polymerisation kinetic parameters definition, which facilitates the system of balance equations to be solved.

\section{The used model}

Literature data [1] show that in real systems, very often during the course of polymerisation processes, the viscosity of the reaction medium changes. When the viscosity changes significantly influence the polymerisation process rate, it becomes 
necessary to take into account these variations in rate equations describing this process. A change in the viscosity of a reaction system, most often exhibiting itself by a change in the numeric kinetic value (which determine rate of reaction), causes a reduction in the reaction rate numerical constants, which should be taken into consideration in numeric calculations. Changes in viscosity, during the course of a polymerisation process, cause a change in the process kinetic parameters, resulting in adding significant complexity to the mass balance equation system. As reaction rate constant values change with the progress of the polymerisation process, it becomes necessary, from the point of view of numeric calculations, to determine the reaction rate values at each step of the said calculations.

This paper uses the mass balance model for the heteropolyaddition process presented in the paper [1]. The model is based on the assumption of a variable reaction rate of the polymerisation process, which results from a change in the numerical values of the kinetic parameters of the process, which is related to the change in the number-average molar mass of the reaction system and the change in the mixture viscosity [2]. Changes in viscosity during the polymerisation process modify the kinetic parameters of the process, making the system of mass balance equations significantly more complex. The values of reaction rate constants vary as the polymerisation process progresses, which means that in numerical calculations they have to be determined at every step of the process. The aforementioned paper assumes that, for a secondary reaction, the rate of which is presented in the form of $r_{i}=k_{\mathrm{AB}} C_{\mathrm{A}} C_{\mathrm{B}}$, a constant reaction rate is $k_{\mathrm{AB}}$. This value is related to the reaction, not to the reactant. In order to associate the reaction rate with the reactant rather than with the reaction, a partial reaction rate constant was used, which is defined as follows:

$$
k_{\mathrm{AB}}=k_{\mathrm{A}} k_{\mathrm{B}} \text {. }
$$

The paper [1] uses a mass balance model based on the reaction of diphenyl carbonate and diglycidyl ether of bisphenol $\mathrm{A}$ in a batch reactor with ideal mixing at $100{ }^{\circ} \mathrm{C}$ for $48 \mathrm{~h}$ to determine the kinetic parameters of the polymerisation process. As in the cited paper, for calculations, this paper uses the system of mass balance equations for the heteropolyaddition process of an alternating linear polymer in a batch reactor with ideal mixing, working in isothermal conditions defined as follows:

$$
\left\{\begin{array}{l}
\frac{\mathrm{d} C_{1}}{\mathrm{~d} t}=-k_{1} C_{1}\left(S_{\mathrm{A}}+S_{\mathrm{AB}}\right), \\
\frac{\mathrm{d} C_{n+1}}{\mathrm{~d} t}=-k_{n+1} C_{n+1}\left(S_{\mathrm{B}}+S_{\mathrm{AB}}\right), \\
\frac{\mathrm{d} C_{2 n+1}}{\mathrm{~d} t}=k_{1} C_{1} k_{n+1} C_{n+1}-k_{2 n+1} C_{2 n+1}\left(S_{\mathrm{A}}+S_{\mathrm{B}}+S_{\mathrm{AB}}\right), \\
\frac{\mathrm{d} C_{p}}{\mathrm{~d} t}=\sum_{i=1}^{p-1} k_{i} C_{i} k_{2 n+p-i} C_{2 n+p-i}-k_{p} C_{p}\left(S_{\mathrm{A}}+S_{\mathrm{AB}}\right), \\
\frac{\mathrm{d} C_{n+p}}{\mathrm{~d} t}=\sum_{i=1}^{p-1} k_{n+i} C_{n+i} k_{2 n+p-i} C_{2 n+p-i}-k_{n+p} C_{n+p}\left(S_{\mathrm{B}}+S_{\mathrm{AB}}\right), \\
\frac{\mathrm{d} C_{2 n+p}}{\mathrm{~d} t}=\sum_{i=1}^{p-1} k_{i} C_{i} k_{n+p+1-i} C_{n+p+1-i}+\sum_{i=1} \quad \text { floor }\left(\frac{p}{2}\right) \\
2 n{ }_{2 n+i} C_{2 n+i} k_{2 n+p-i} C_{2 n+p-i}-k_{2 n+p} C_{2 n+p}\left(S_{\mathrm{A}}+S_{\mathrm{B}}+S_{\mathrm{AB}}\right)
\end{array}\right.
$$


The above system of mass balance was based on the model of aggregation presented in the study [3]. Then, the model presented in the study [4, 5] was modified to obtain the model of mass balance (2). This paper presents research that employs model (2) based on the process of linear polyurethane formation resulting from the heteropolyaddition of toluene-2,4-diisocyanate (TDI) and 1,4-butanediol (BD) in dilute solution [6]. The heteropolyaddition process was based on the reaction of isocyanate with a $-\mathrm{OH}$ hydroxyl group compound, in this case with alcohol. The general chemical equation can be represented as [7]:

$$
\underset{\mathrm{A}}{\mathrm{R}_{1}-\mathrm{NCO}}+\underset{\mathrm{B}}{\mathrm{R}_{2}-\mathrm{OH}} \longrightarrow k_{\mathrm{AB}} \begin{gathered}
\mathrm{R}_{1}-\mathrm{NH}-\mathrm{CO}-\mathrm{OR}_{2} \\
\mathrm{AB}
\end{gathered}
$$

In order to make it easier to associate reactants with the used model (2), toluene-2,4-diisocyanate was marked with symbol A and 1,4-butanediol with symbol $B$. Heteropolyaddition process modelling was performed on the basis of model (2) and laboratory tests carried out in the paper [7]. The authors of this paper focused entirely on the completed research presented in the paper [7]. The heteropolyaddition process was carried out in a batch reactor with ideal mixing at a constant temperature of $86{ }^{\circ} \mathrm{C}$ in a solution of chlorobenzene $\left(\mathrm{C}_{6} \mathrm{H}_{5} \mathrm{Cl}\right)$ and tetrahydrofuran (THF) at a mass ratio of $1: 1$. The prepared reaction mixture consisted of $30 \mathrm{~g}$ of toluene-2,4-diisocyanate $(0.172 \mathrm{~mol})$ and $15.5 \mathrm{~g}$ of 1,4 -butanediol $(0.172 \mathrm{~mol})$ dissolved in $409.5 \mathrm{~g}$ of chlorobenzene and tetrahydrofuran solution. Due to the $10 \%$ concentration of the reactants, the analysed example of the reaction solution should be considered as diluted solution. The heteropolyaddition process was analysed for 300 min during which the authors of [7] took samples at different intervals in order to determine the concentration of free functional groups -NCO using the GPC method. The results of the experimental studies are summarised in Table 1.

The symbols in Table 1 specify, respectively:

- $F 1$ - fraction 1 is equal to the sum of the concentration of components A and B.

- $F 2$-fraction 2 is equal to the concentration of component AB.

Table 1 The mass ratios and number-average molar masses $M_{n}[\mathrm{~g} / \mathrm{mol}]$ of the selected fractions of the reaction mixture in the heteropolyaddition process in 2,4-TDI $(0.172 \mathrm{~mol})$ and $1,4-\mathrm{BD}(0.172 \mathrm{~mol})$ in the solution of chlorobenzene and tetrahydrofuran at a temperature of $t_{1}=86{ }^{\circ} \mathrm{C}$ [7]

\begin{tabular}{lllll}
\hline$t$ [min $]$ & $M_{n}[\mathrm{~g} / \mathrm{mol}]$ & $\begin{array}{l}F 1+F 2 \\
\text { Mass proportion }\end{array}$ & $F 3+F 4$ & $F 5+F 6$ \\
\hline 20 & 309 & 0.73 & 0.252 & 0.016 \\
40 & 332 & 0.574 & 0.352 & 0.074 \\
60 & 356 & 0.46 & 0.385 & 0.155 \\
90 & 412 & 0.32 & 0.354 & 0.225 \\
120 & 456 & 0.252 & 0.314 & 0.267 \\
180 & 537 & 0.164 & 0.234 & 0.269 \\
240 & 667 & 0.087 & 0.157 & 0.218 \\
300 & 801 & 0.067 & 0.112 & 0.166 \\
\hline
\end{tabular}


- $F 3$ - fraction 3 is equal to the sum of the concentration of components $\mathrm{A}_{1} \mathrm{~B}_{2}$ and $\mathrm{A}_{2} \mathrm{~B}_{1}$.

- $F 4$-fraction 4 is equal to the concentration of component $\mathrm{A}_{2} \mathrm{~B}_{2}$.

- $F 5$-fraction 5 is equal to the sum of the concentration of components $\mathrm{A}_{2} \mathrm{~B}_{3}$ and $\mathrm{A}_{3} \mathrm{~B}_{2}$.

- $F 6$ - fraction 6 is equal to the concentration of component $\mathrm{A}_{3} \mathrm{~B}_{3}$.

The numerical calculations of the presented heteropolyaddition process were based on model (2), on the basis of which a calculation algorithm was built in the Mathcad Prime 2.0 software that allows for approximation of kinetic parameters of the process and consequently for determining the kinetics of the polyurethane-forming reaction.

The calculations were based on the results of experimental studies in Table 1 and the model of the heteropolyaddition process (2). All calculations were done starting with determining the reaction rate constant $k_{\mathrm{AB}}=5 \times 10^{-4} \mathrm{dm}^{3} /(\mathrm{mol} \mathrm{s})$ with the assumption $k_{i}=$ const.

In the calculations, the correlations describing the partial reaction rate constant $k_{i}$ and the correction coefficient $Z_{i}$ presented in [1] were used in an analogous manner. The correlation describing a partial reaction rate constant $k_{i}$ is presented in the form of:

$$
k_{i}=k_{1} \cdot Z_{i}\left(M_{n}\right)
$$

The correction coefficient $Z_{i}$ in Eq. (3) is a function of the number-average molecular mass of the reaction mixture. In order to determine the function of $Z_{i}\left(M_{n}\right)$, the correlation of $Z_{i}$ correction coefficient was used:

$$
Z_{i}\left(M_{n}\right)=\gamma_{1} \cdot M_{n}^{-p},
$$

where $\gamma_{1}=\gamma \cdot M_{0}^{p} ; M_{0}$-molecular mass of monomer A (or B)

Equation (4) defines the correction coefficient, which is a dimensionless number, with two parameters $\gamma_{1}$ and $p$ characteristic for a particular polymerisation process. Due to this fact, the partial reaction rate constant of $k_{i}$ decreases with the increase in the number-average molar mass of the forming polymer.

Estimation of kinetic parameters (particle reaction rate constants) $k_{i}$ was performed on the basis of results of experimental research using numerical method of simplex optimisation. The simplex optimisation method minimised the value of the objective function $S$, determined by the sum of squares of differences in the calculated $O_{i}$ values and experimental $E_{i}$ values. The optimum numerical value of this constant was determined by minimising the numerical value of the objective function, defined by the equation:

$$
S=\sum_{i=1}^{b}\left(O_{i}-E_{i}\right)^{2} \rightarrow \min .
$$

As a result of the calculations done, parameters $p=0.01$ and $\gamma_{1}=1$ were obtained from Eq. (4), which then allowed to estimate values of the partial reaction rate constants, as summarised in Table 2. 
Table 2 Selected values of the partial reaction rate constant $k_{i}\left[\left(\mathrm{dm}^{3} /(\mathrm{mol} \mathrm{s})\right)^{1 / 2}\right]$ in the heteropolyaddition process in 2,4-TDI (0.172 mol) and 1,4-BD $(0.172 \mathrm{~mol})$ in chlorobenzene and tetrahydrofuran solution at temperature $t_{1}=86^{\circ} \mathrm{C}$ are calculated with model (2)

\begin{tabular}{ll}
\hline$t[\mathrm{~min}]$ & $k_{i}\left[\left(\mathrm{dm}^{3} /(\mathrm{mol} \mathrm{s})\right)^{1 / 2}\right]$ \\
\hline 0 & 0.02020 \\
20 & 0.02015 \\
40 & 0.02010 \\
60 & 0.02006 \\
90 & 0.02001 \\
120 & 0.01997 \\
180 & 0.01991 \\
240 & 0.01986 \\
300 & 0.01982 \\
\hline
\end{tabular}

Table 3 Comparison of experimental data and the data calculated with model (2) in the form of mass ratios and number-average molar mass $M_{n}[\mathrm{~g} / \mathrm{mol}]$ of the selected fractions of the reaction mixture in the heteropolyaddition process in 2,4-TDI $(0.172 \mathrm{~mol})$ and $1,4-\mathrm{BD}(0.172 \mathrm{~mol})$ in the solution of chlorobenzene and tetrahydrofuran at a temperature of $t_{1}=86^{\circ} \mathrm{C}[7]$

\begin{tabular}{|c|c|c|c|c|c|c|c|c|}
\hline \multirow[t]{3}{*}{$t[\min ]$} & \multicolumn{4}{|c|}{ Data from literature [7] } & \multicolumn{4}{|c|}{ Calculated with model (2) } \\
\hline & \multirow[t]{2}{*}{$M_{n}[\mathrm{~g} / \mathrm{mol}]$} & \multicolumn{3}{|l|}{$w_{i}$} & \multirow[t]{2}{*}{$M_{n}[\mathrm{~g} / \mathrm{mol}]$} & \multicolumn{3}{|l|}{$w_{i}$} \\
\hline & & $F 1+F 2$ & $F 3+F 4$ & $F 5+F 6$ & & $F 1+F 2$ & $F 3+F 4$ & $F 5+F 6$ \\
\hline 20 & 309 & 0.730 & 0.252 & 0.016 & 166 & 0.818 & 0.162 & 0.018 \\
\hline 40 & 332 & 0.574 & 0.352 & 0.074 & 207 & 0.590 & 0.294 & 0.087 \\
\hline 60 & 356 & 0.460 & 0.385 & 0.155 & 250 & 0.430 & 0.326 & 0.150 \\
\hline 90 & 412 & 0.32 & 0.354 & 0.225 & 316 & 0.284 & 0.300 & 0.195 \\
\hline 120 & 456 & 0.252 & 0.314 & 0.267 & 383 & 0.200 & 0.253 & 0.200 \\
\hline 180 & 537 & 0.164 & 0.234 & 0.269 & 519 & 0.114 & 0.176 & 0.171 \\
\hline 240 & 667 & 0.087 & 0.157 & 0.218 & 655 & 0.074 & 0.126 & 0.137 \\
\hline 300 & 801 & 0.067 & 0.112 & 0.166 & 792 & 0.052 & 0.094 & 0.110 \\
\hline \multicolumn{9}{|c|}{ The value of objective function $\mathrm{S}$ in accordance with Eq. (5) } \\
\hline & $S_{M_{n}}$ & $S_{F 1+F 2}$ & $S_{F 3+F 4}$ & $S_{F 5+F 6}$ & $S_{M_{n}}$ & $S_{F 1+F 2}$ & $S_{F 3+F 4}$ & $S_{F 5+F 6}$ \\
\hline$S$ & 185,000 & 0.0480 & 0.0451 & 0.0457 & 62,370 & 0.0188 & 0.0274 & 0.0201 \\
\hline$\Sigma S$ & - & 0.1388 & & & - & 0.0623 & & \\
\hline
\end{tabular}

Determination of kinetic parameters for two different $k_{i}$ values, $k_{i}=$ const [8] and $k_{i}=k_{1} Z_{i}$, allowed to determine how the mass ratios of respective polymer fractions change with time, and compare them with the results of experimental research, as shown in the figures below.

The results of model (2) research are summarised in Table 3 and compared with the experimental data.

The points in Figs. 1, 2, 3, 4, 5, 6, 7 and 8 represent the correlations obtained from the literature [7] from experimental research, while the continuous lines represent the correlations calculated using model (2) in two cases: 


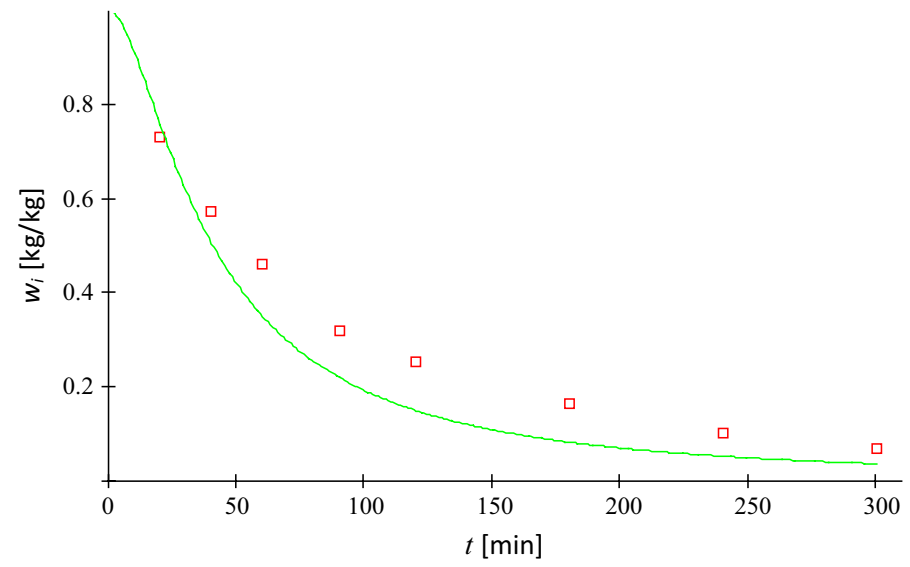

Fig. 1 Change in the mass ratio of polyurethane fraction $F 1$ over time, $k_{i}=$ const; line calculated with model (2), box experimental data [7]

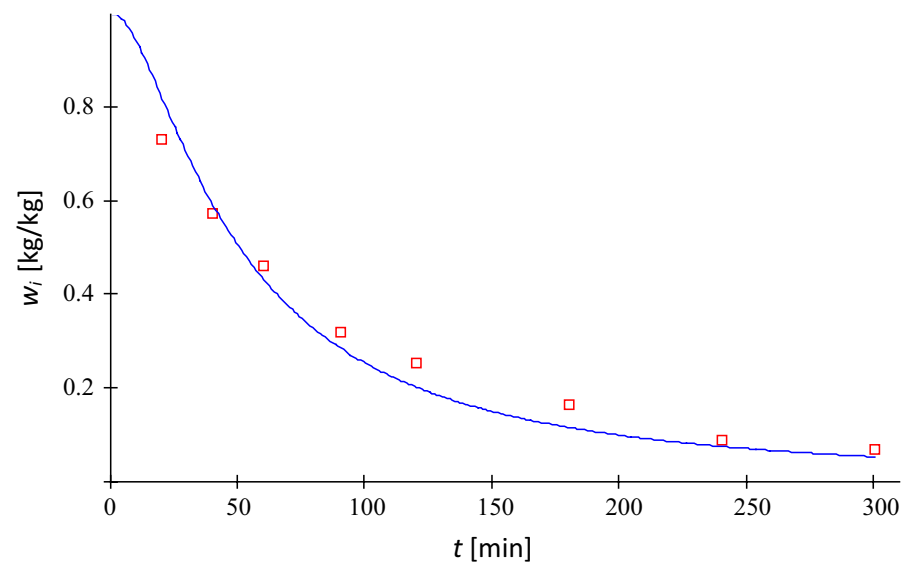

Fig. 2 Change in the mass ratio of polyurethane fraction $F 1$ over time, $k_{i}=k_{1} Z_{i}$; line calculated with model (2), box experimental data [7]

- at the same value of reaction rate constant $k_{i}=$ const

- with a reaction rate constant dependent on the average molecular mass of the polymer $k_{i}=k_{1} Z_{i}$.

On the basis of Figs. 1, 2, 3, 4, 5, 6, 7 and 8 and data in Table 3, it can be concluded that if the increase in viscosity of the reaction mixture is omitted and the same reaction rate is assumed during the whole process of heteropolyaddition of 2,4-TDI with 1,4-BD, the result obtained from the sum of objective function (5) for all analysed fractions $S_{\min }=0.1388$ is twice higher than when including the 


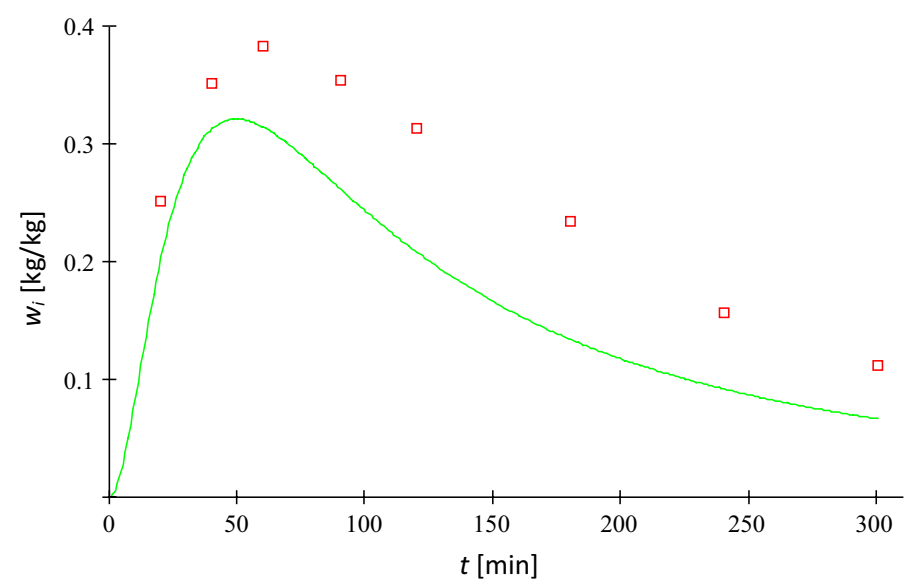

Fig. 3 Change in the mass ratio of polyurethane fraction $F 2$ over time, $k_{i}=$ const; line calculated with model (2), box experimental data [7]

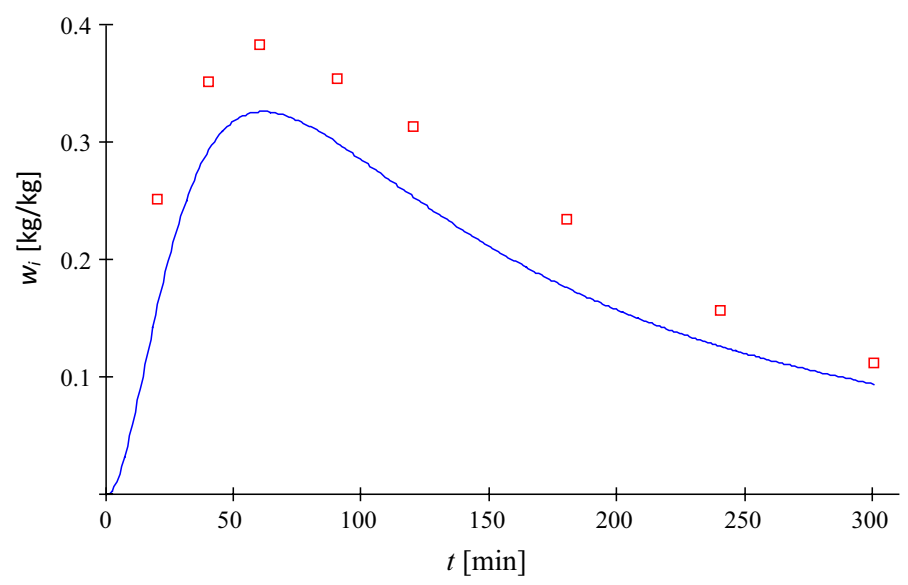

Fig. 4 Change in the mass ratio of polyurethane fraction $F 2$ over time, $k_{i}=k_{1} Z_{i}$; line calculated with model (2), box experimental data [7]

changes in the average molecular mass of the forming polymer (and thus viscosity) $S_{\min }=0.0623$.

In order to compare the results obtained in Table 3 , an additional correlation coefficient $\beta(\eta)$ was introduced, which takes into account the influence of viscosity on the polymerisation process and is expressed in the form of an equation:

$$
\beta(\eta)=\frac{S_{C}-S_{\eta}}{S_{C}} .
$$




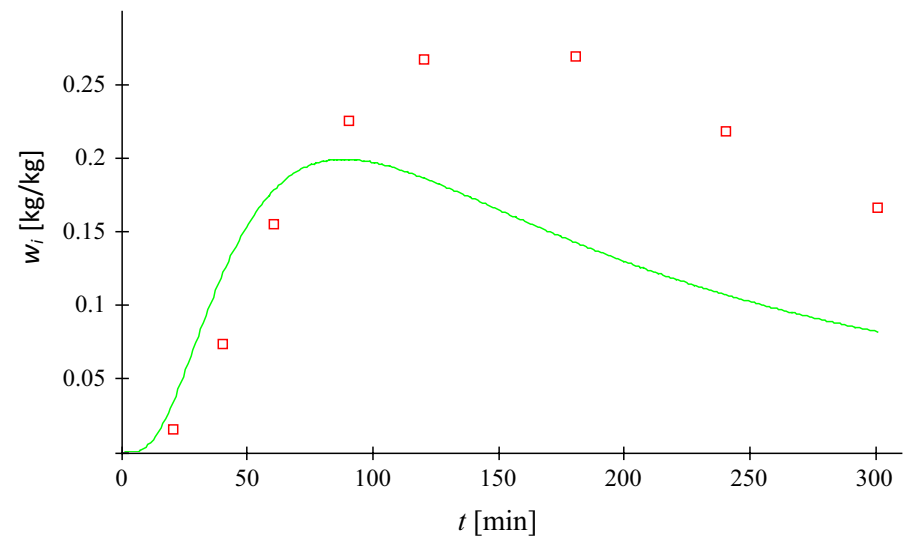

Fig. 5 Change in the mass ratio of polyurethane fraction $F 3$ over time, $k_{i}=$ const; line calculated with model (2), box experimental data [7]

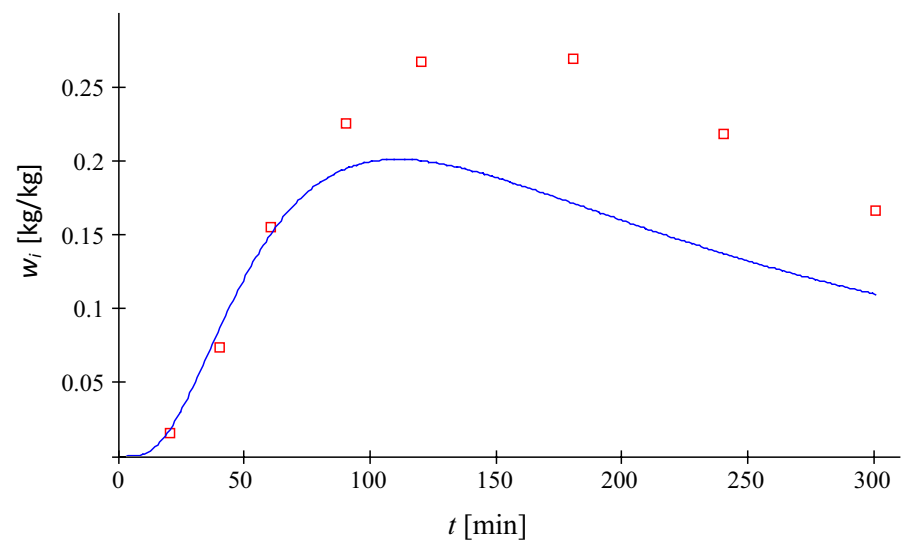

Fig. 6 Change in the mass ratio of polyurethane fraction $F 3$ over time, $k_{i}=k_{1} Z_{i}$; line calculated with model (2), box experimental data [7]

In the above equation, the following symbols were used: $S_{\mathrm{c}}$-results of calculations without changes in viscosity; $S_{\eta}$-results of calculations with changes in viscosity; and $\beta(\eta)$-numerical value of correlation coefficient included in the range $(0,1): \beta(\eta) \in(0,1)$.

Using Eq. (6) and the results compiled in Table 3, a correlation factor $\beta(\eta)$ has been calculated for the theoretical and model (2) values, with the following results:

$$
\beta_{t}(\eta)=\frac{0.1388-0.0623}{0.1388}=0.551
$$

The measure of the influence of viscosity on the polymerisation process in this case is the value of the correlation coefficient $\beta(\eta)$ which increases with the 


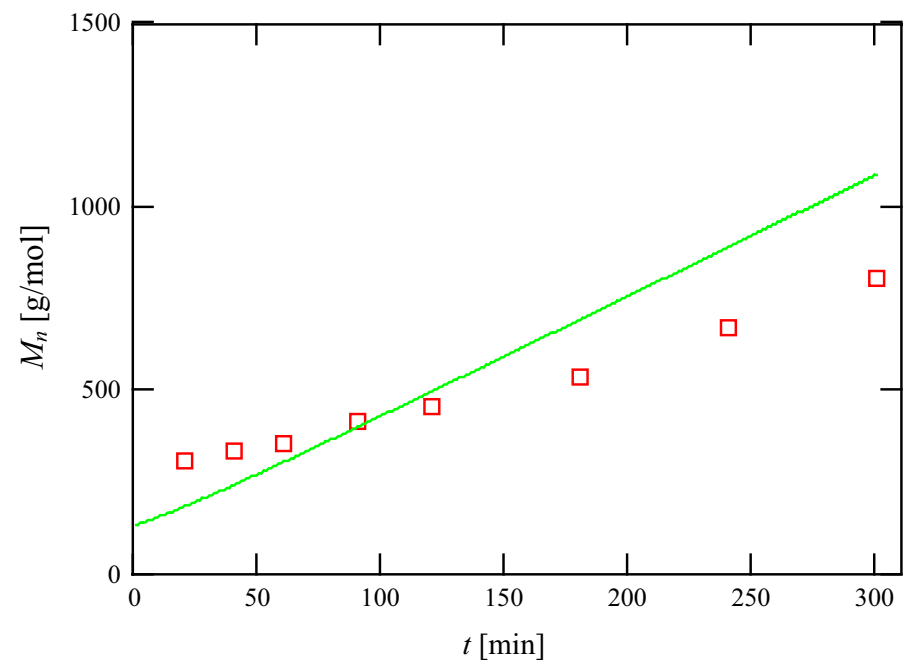

Fig. 7 Correlation of number-average molar mass $M_{n}[\mathrm{~g} / \mathrm{mol}]$ over time in the heteropolyaddition process in 2,4 -TDI $(0.172 \mathrm{~mol})$ and $1,4-\mathrm{BD}(0.172 \mathrm{~mol})$ in the chlorobenzene and tetrahydrofuran solution at temperature $t_{1}=86^{\circ} \mathrm{C}, k_{i}=$ const; line calculated with model (2), box experimental data [7]

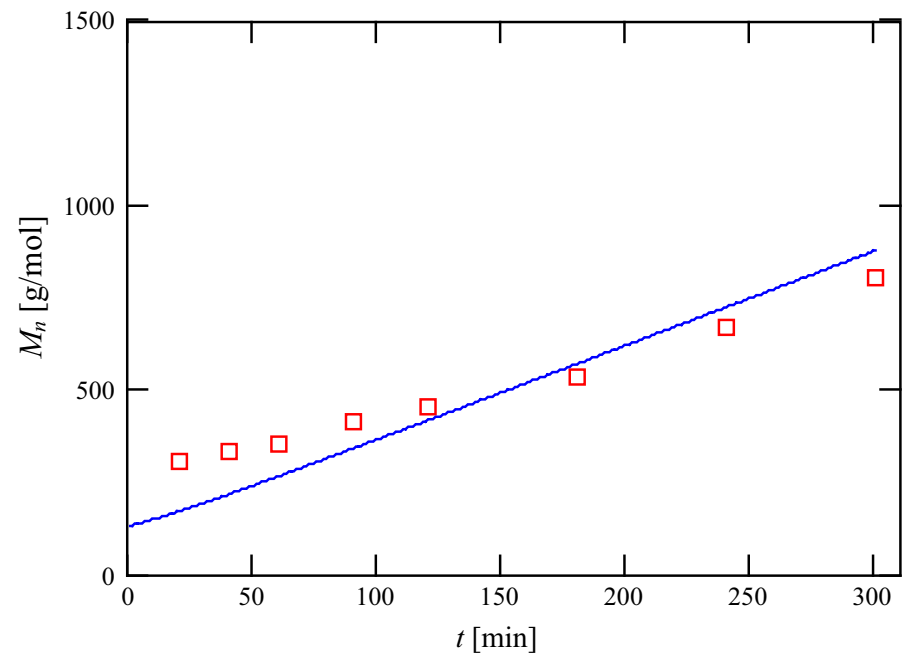

Fig. 8 Correlation of number-average molar mass $M_{n}[\mathrm{~g} / \mathrm{mol}]$ over time in the heteropolyaddition process in 2,4-TDI $(0.172 \mathrm{~mol})$ and $1,4-\mathrm{BD}(0.172 \mathrm{~mol})$ in the chlorobenzene and tetrahydrofuran solution at temperature $t_{1}=86{ }^{\circ} \mathrm{C}, k_{i}=k_{1} Z_{i}$; line calculated with model (2), box experimental data [7]

increase in viscosity influence on the process. The value of this coefficient was $\beta_{t}(\eta)=0.551$. The figure below shows the dynamics of changes in the correction coefficient $Z_{i}$ in relation to the average molar mass. 
It is worth noting that this case is an example of a polymerisation in diluted solutions, as evidenced by a small change in the $Z_{i}$ correction coefficient, varying between 0.9530 and 0.9550 .

\section{Conclusions}

Comparing the results of polyurethane formation process modelling with the results obtained in the studies [1, 4], it should be emphasised that the proposed method of determining kinetic parameters is rather reserved for cases where the viscosity of the reaction solution has a significant influence on the speed of the polymerisation process. The viscosity influence on the rate of heteropolyaddition process is determined by the correction coefficient $Z_{i}$ related to the average molar mass of the forming polymer. It should be noted that the $Z_{i}$ correction coefficient in the analysed process of heteropolyaddition of chloride 1,4-benzene dicarbonate with diglycidyl ether of bisphenol $\mathrm{A}$ is in the range of 1.6-0.2 [1], while that in the heteropolyaddition of 2,4-TDI with 1,4-BD is in the range of 0.9530-0.9550 (Fig. 9), which indicates that there is a significant influence of viscosity on the process in the first case. Despite the high dilution of the reaction mixture and slight change in viscosity of the system during the process, it can be observed that the proposed method gives better results in clarifying the process.

This paper deliberately analyses a process in a solution in which the viscosity is not likely to affect the speed of the polymerisation process or its influence is negligible. The aim was to examine how model (2) behaves, assuming a variable value of

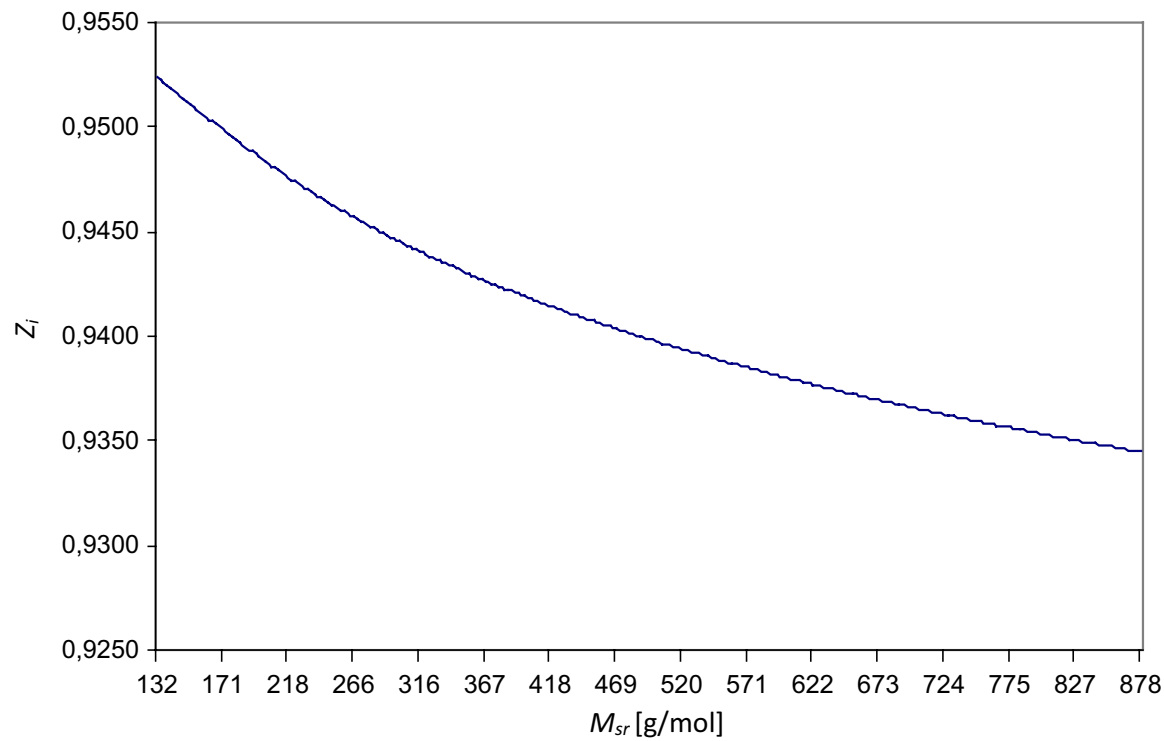

Fig. 9 Change in $Z_{i}$ correction coefficient in the average molar mass $M_{\mathrm{sr}}[\mathrm{g} / \mathrm{mol}]$ of the forming polyurethane at a temperature of $86{ }^{\circ} \mathrm{C}$ calculated with model (2) 
kinetic parameters. As we can see, despite slight changes in viscosity of the system resulting from a small increase in average molar mass of polyurethane, the proposed method of kinetic parameters calculation gives a better approximation of the course of polyaddition process of 2,4-TDI with 1,4-BD than the model based on the same kinetic parameters for the whole polymerisation process.

The results obtained show that the applied approach proposed in model (2) is universal, and prove that kinetic parameters change during the heteropolyaddition process.

Open Access This article is distributed under the terms of the Creative Commons Attribution 4.0 International License (http://creativecommons.org/licenses/by/4.0/), which permits unrestricted use, distribution, and reproduction in any medium, provided you give appropriate credit to the original author(s) and the source, provide a link to the Creative Commons license, and indicate if changes were made.

\section{References}

1. Gawdzik A, Mederska A, Mederski T (2017) Modelling polycarbonate synthesis rates on the example of bulk heteropolyaddition of diphenyl carbonate and bisphenol a diglycidyl ether. Polym Bull 74(5):1553-1571

2. Chinai SN (1957) Calculation of molecular weight and dimensions of polymers from viscosity interaction parameter. Ind Eng Chem 49(2):303-304. https://doi.org/10.1021/ie50566a050

3. Smoluchowski M (1917) Versuch einer mathematischen Theorie der Koagulationskinetik kolloider Lösungen. Z Phys Chem 92:129-168

4. Gawdzik A, Mederska A, Mederski T (2012) Effect of viscosity changes of reaction mixture on the kinetics of formation of linear living polymer. Ecol Chem Eng A 19(11):1393-1403. https://doi. org/10.2428/ecea.2012.19(11)135

5. Król P, Gawdzik A (1995) Basic kinetic model for the reaction yielding linear polyurethanes. II. J Appl Polym 58(4):729-743. https://doi.org/10.1002/app.1995.070580406

6. Król P, Heneczkowski M (2000) Numerical simulation of polymerization processes of diisocyanates and diols. Polimery 45(3):161-171

7. Król P (1995) Studia na kinetyka reakcji otrzymywania liniowych poliuretanów. Habilitation dissertations No. 2922, Jagiellonian University

8. Flory PJ (1953) Principles of polymer chemistry. Cornell University Press, Ithaca 